\title{
Planificación urbana, regeneración urbana integral y participación ciudadana en Cataluña: balance y retos de futuro
}

\section{David Mongil Juárez*}

\section{Resumen}

Se analiza la incorporación de la participación ciudadana en los instrumentos de planificación urbana y de regeneración urbana integral de Cataluña a lo largo de los últimos años. En este sentido, se aborda el análisis de la participación ciudadana y su papel en el fomento de una nueva cultura del urbanismo, y se realiza una valoración del estado de la participación ciudadana en los instrumentos de planificación urbanística y de regeneración urbana integrada en Cataluña, que puede sintetizarse en:

- El significativo, aunque mejorable, avance en la incorporación de la participación ciudadana a la normativa que regula los instrumentos de planificación urbanística y de rehabilitación urbana integrada.

- La progresiva, aunque incipiente, evolución de los modelos de participación ciudadana aplicados a los iniciativas urbanísticas.

- La limitada influencia de los procesos participativos en los procesos de elaboración y de toma de decisiones de los instrumentos de planificación urbanística y de regeneración urbana integral.

\section{Palabras clave}

Planeamiento Urbanístico; Rehabilitación Urbana Integral; Participación Ciudadana; Cataluña; Balance; Retos

\section{Abstract: Urban planning, urban regeneration and citizen participation in Catalonia: outcomes and challenges}

The aim of the article is about the citizen participation in Catalonia's urban planning and urban regenerations. At this sense, the text analyse citizen participation and new urban planning culture, and evaluate citizen's participation in CataIonia's urban planning and urban regeneration This evaluation is summed up in:

- The progress in the rules and applications of citizen participation about urban planning, and urban regeneration.

- The progressive evolution of citizen participation model's in urban planning, and urban regeneration.

- The limited influence of citizen participation in urban planning and urban regeneration decision-making process.

\section{Key words}

Urban Planning; Urban Regeneration; Citizen Participation; Catalonia; Outcomes; Challenges

\footnotetext{
Recibido: 16/07/2012; aceptado definitivamente: 16/07/2012

* Licenciado en Geografía y Especialista Universitario en Ordenación del Territorio y Medio Ambiente. Servicio de Planificación de Vivienda y Mejora Urbana de la Gerencia de Servicios de Vivienda, Urbanismo y Actividades de la Diputación de Barcelona. Vicepresidente del Colegio de Geógrafos de España. Dirección de contacto: C/ Aragón 369, 2o 1a. CP. 08009 Barcelona (Barcelona). E-mail: mongiljd@diba.cat

(Las opiniones y reflexiones recogidas en este artículo pertenecen exclusivamente a su autor y no pueden hacerse extensivas a la institución en que desarrolla su labor profesional ni al colectivo profesional al que representa).
} 


\section{Introducción}

A lo largo de los últimos años se ha asistido a un significativo impulso y a una creciente demanda de un urbanismo más participativo, es decir, en el que el papel de la ciudadanía en la definición, ejecución y seguimiento de las iniciativas de intervención en la ciudad fuese más importante. Dicha situación ha tenido su traslación en la práctica urbanística de muchos territorios.

En este sentido, el presente artículo tiene como objeto el análisis y valoración de la incorporación y tratamiento de la participación ciudadana en el urbanismo catalán a lo largo de la última década, desde 2002 (fecha en la que se aprobó la primera ley de urbanismo catalana que recogía como una de sus principios rectores "la publicidad y participación en los procesos de planificación y gestión urbanística") hasta el momento actual (junio de 2012), analizando la evolución de la normativa ligada a la intervención urbana, las formas y modelos de aplicación de la participación ciudadana, y sus resultados y retos de futuro. Consecuentemente, el texto se articula en tres apartados:

- En el primero, a partir de la bibliografía existente, se realiza una aproximación a la noción de participación ciudadana, y especialmente al papel que ésta puede jugar en el refuerzo de una nueva (o diferente) cultura del urbanismo basada en la valorización de sus valores sociales y ambientales.

- En el segundo, a partir de los datos y publicaciones existentes, especialmente de los publicados por la administración autonómica, se procede al análisis de la incorporación de la participación ciudadana en los principales marcos legales-instrumentales ligados al urbanismo en que se ha manifestado en
Cataluña: la planificación urbanística y la regeneración urbana integral ${ }^{1}$.

- En el tercero, a partir de los datos existentes, de la experiencia y resultados prácticos conocidos por el autor, y de la puesta en relación de estas dos cuestiones con la bibliografía existente, se procede a valorar el estado actual de la participación ciudadana en el urbanismo catalán y se apuntan posibles elementos de mejora.

\section{La participación ciudadana y su papel en el fomento de una nueva cultura del urbanismo}

La rapidez de las transformaciones económicas, socioculturales y ambientales de los últimos años está dando lugar a significativos cambios en nuestra sociedad. Cambios que tienen un efecto directo sobre las administraciones, que han de afrontar sus retos y responsabilidades en un contexto de creciente complejidad e incertidumbre en el que la desconfianza y desafección ciudadana crecen (Sampedro y Taibo, 2006; Subirats, 2011; Taibo, 2011). Ante de esta situación, surge la necesidad de hacer política pública de otra manera, basada en los principios de una gobernanza donde la concertación, la cooperación y la participación constituyan algunos de sus principios básicos (Bobbio, 2004; Farinós, 2005; Subirats, 2006 y 2009; Romero, 2006; Farinós y Romero, 2007; Brugué, 2009).

Así, a lo largo de los últimos años, hemos asistido a un proceso de consolidación conceptual de una "Participación Ciudadana" dirigida a favorecer una democracia más deliberativa y colaborativa (Habermas, 1984; Giddens, 1984; Healy, 1997), y

\footnotetext{
${ }^{1}$ Cabe reseñar que si bien el autor parte de una concepción amplia del urbanismo, y de que por tanto hubiera cabido la posibilidad de analizar igualmente otro tipo de iniciativas e instrumentos (como por ejemplo los planes municipales de vivienda, o los planes de movilidad), la extensión máxima permitida para el texto nos ha obligado a centrar el análisis en
} 
manifestada en forma de política pública (entendida dentro de los límites de la democracia representativa) dirigida a favorecer la influencia, el sentimiento de pertenencia, la corresponsabilización y la capitalización del conocimiento de la ciudadanía en los procesos de definición, implantación y evaluación de la acción pública (Villasante, 1995; Marchioni, 1999; Pindado, 1999 y 2009; Martí y Rebollo, 2005; Font y Blanco, 2006; Brugué, 2009; Subirats, 2009).

Sobre esta base teórica y conceptual, en el caso de Cataluña, desde 2003 se ha asistido, no ya sólo a la incorporación de la participación ciudadana a la normativa institucional básica de referencia, es decir al Estatuto de Autonomía ${ }^{2}$, sino también a la configuración de una política específica de participación ciudadana que, tal y como muestran los datos e informaciones de las memorias y publicaciones editados por la Generalitat de Catalunya (Generalitat de Catalunya, 2010; Font i Galais, 2009), se ha manifestado en:

- La visualización de la participación ciudadana en los organigramas de los gobiernos autonómicos y municipales. Así, en 2008, el $54,8 \%$ de los 93 municipios en que se habían detectado y desarrollado experiencias participativas contaban con una concejalía de participación (específica o compartida con otras materias).

- Una significativa inversión pública para el desarrollo de la política pública de participación ciudadana. Así, entre 2005 y 2010, el importe de las ayudas concedidas por la Generalitat de Catalunya a los ayuntamientos (sin contar las aportaciones

\footnotetext{
la planificación urbanística y la regeneración urbana integral.

2 En este sentido, el Estatuto de Autonomía de Cataluña identifica dentro de los principios rectores de los poderes públicos (con el potencial y los límites que ello conlleva) el del Fomento de la Participación, manifestado en "la obligación de los poderes públicos de promover la participación social en la elaboración, prestación y evaluación de las políticas públicas".
}

propias aportadas por los mismos) alcanzó los 10.069.722 euros.

- La profesionalización de la participación ciudadana, manifestada en la creciente aparición de técnicos especializados en la materia tanto en el sector público como en el privado.

- Su carácter de iniciativa pública y abierta. Así, en diciembre de 2009 , el $80,6 \%$ de las iniciativas participativas se habían desarrollado por iniciativa exclusiva de los gobiernos locales, en un $61,2 \%$ de los casos de forma abierta al conjunto de la ciudadanía.

- La extensión de prácticas participativas a todo tipo de territorios y colores políticos de los equipos de gobierno, si bien ha tenido mayor calado y desarrollo en los municipios de mayor población, en la región metropolitana barcelonesa y en gobiernos de centroizquierda. En todo caso, en 2008 hasta un $30,1 \%$ de las experiencias participativas que se habían identificado se localizaban en ayuntamientos inferiores a los 10.000 habitantes, y entre un $18 \%$ y un $22 \%$ de ellas se habían realizado en ayuntamientos con equipos de gobierno conformados exclusivamente por grupos políticos considerados de derechas.

- Su naturaleza mayoritariamente consultiva (70,9 \% de las experiencias detectadas en 2009) y, en menor medida, al co-diseño (14,6 $\%$ ), la co-decisión (8,7\%) y la co-gestión $(5,5 \%)$.

- Su concreción diversa en formas (órganos de participación estable, procesos y mecanismos puntuales), fases (diagnosis, programación, decisión o implementación), metodologías (talleres, asambleas o reuniones abiertas, metaplanes, cuestionarios, grupos de discusión, etc.) y temáticas (tanto específicamente dirigidas a la política 
municipal general o de participación ciudadana en particular, como a la aplicación de la participación ciudadana en iniciativas de otras políticas sectoriales) ${ }^{3}$.

Precisamente los datos de distribución temática de las iniciativas participativas muestran que el urbanismo, entendido en un sentido amplio del término y no exclusivamente por su derivada instrumental de mayor calado y reconocimiento, el planeamiento urbanístico ${ }^{4}$, ha sido la principal cuestión sobre la que se han aplicado iniciativas participativas. Así, a modo de ejemplo basta apuntar que una tercera parte (concretamente un $34 \%$ ) del total de iniciativas participativas locales desarrolladas entre 2004 y 2009 estuvieran relacionadas con el urbanismo, y que un $22 \%$ de las ayudas prestadas por la antigua Dirección General de Participación Ciudadana de la Generalitat de Catalunya durante el período 2005-2010 fueron dirigidas a iniciativas urbanísticas y territoriales.

Los motivos de esta interrelación entre urbanismo y participación tienen que ver con la propia configuración del urbanismo como una de los temas clave de la política pública, tanto por su función social como por su capacidad de generar beneficios económicos a través de los procesos de transformación del suelo, y en la que, precisa-

\footnotetext{
${ }^{3}$ Toda aquellas persona interesada puede consultar los datos concretos de la diversidad de formas, metodologías y temáticas en los trabajos anteriormente citados.

4 Esta consideración amplia del urbanismo es habitual en las aproximaciones a la materia más académicas y relacionadas con las ciencias sociales, tal y como apunta por ejemplo el Diccionario de geografía urbana, urbanismo y ordenación del territorio (Grupo ADUAR - Varios Autores -. Ed. Ariel. Año 2000) para quien el urbanismo constituye la ciencia encargada de la "reflexión, proyección, construcción y transformación de las ciudades o de alguna de sus partes". Pero también apuntan en la misma línea de concepción amplia del urbanismo y de no confusión entre la ciencia y sus instrumentos, reconocidos especialistas en el campo práctico del planeamiento urbanístico como Juli Esteban, cuando define el urbanismo como "la disciplina que aporta las técnicas a la formulación y el desarrollo de proyectos urbanos colectivos a largo plazo, especialmente mediante los instrumentos de planeamiento, gestión y el mecanismo de disciplina urbanística" en el reconocido manual L'ordenació urbanística: conceptes, eines $i$ pràctiques reeditado por la Diputación de Barcelona en 2007.
}

mente por esta doble naturaleza, se manifiestan con especial fuerza:

- La desafección y desconfianza, sea más o menos justa ${ }^{5}$, de buena parte de la ciudadanía respecto al urbanismo y al papel que la administración juega en el mismo, al que consideran una práctica opaca, condicionada por intereses particulares, y susceptible de posibles corruptelas.

- El potencial de la práctica urbanística como medio de satisfacción de una parte de las demandas y reivindicaciones ciudadanas más comunes (redes y equipamientos, espacio y transporte público, vivienda digna, etc.).

- La propia complejidad no ya sólo del hecho urbano (que requiere de visiones y conocimientos integrales y transversales) sino también de la gestión de la práctica urbanística, que aúna una componente político-estratégico-económica

(potencialmente generadora de significativos ingresos tanto para una administración con una capacidad económica y financiera limitada como para los particulares), con un marco competencial complejo (en el que confluyen con diferente función y rol no ya sólo diversas administraciones sino diferentes departamentos y estructuras de cada una de ellas) y una amplia diversidad de demandas, visiones y necesidades ciudadanas.

Es decir, en la reflexión, proyección, construcción y reconstrucción de la ciudad confluyen el interés general y los intereses particulares, la propiedad pública y la propiedad privada, y la consideración

\footnotetext{
${ }^{5}$ En este sentido, nos gustaría destacar que cabe no confundir la urbanística, disciplina que tiene entre sus fundamentos y finalidades básicas la garantía de la defensa de la función social y del interés general, con las prácticas que algunas personas han realizado al calor de los beneficios económicos que puede aportar la transformación del suelo o la simple especulación sobre el mismo (al efecto se recomienda consultar el Informe: "Urbanismo y democracia. Alternativas para evitar la corrupción" elaborado por la Fundación Alternativas en 2007).
} 
del territorio como un activo económico que cabe capitalizar $^{6}$ y preservar socio-ambientalmente. En este sentido, la participación ciudadana puede ayudar a prevenir conflictos, acercar posturas, generar empatías, reflexionar complejamente y generar consensos (López et. al., 2010; Benabent, 2010; Mongil, 2011)

En consecuencia, en los últimos años se ha asistido en Cataluña (al igual que en otros muchos territorios), a la incorporación de la participación ciudadana en la redacción y ejecución de instrumentos urbanísticos. A continuación nos acercamos a la forma en que se ha abordado dicha cuestión.

\section{Marco normativo y grado de implantación de la participación ciudadana en los instrumentos de planificación urbanística y de regeneración urbana integrada en Cataluña}

\section{La participación ciudadana en los instrumentos de planificación urbanística}

Sobre la base de una consideración simplificada, puramente legalista e instrumental, la normativa catalana, desde la ya pretérita Ley 2/2002 de Urbanismo de Cataluña, hasta la vigente Ley 3/2012 del 22 de febrero, de modificación del texto refundido de la Ley de Urbanismo, equipara los conceptos de urbanismo y de planeamiento urbanístico $^{7}$ y, así, define a este (art..1.2) como la "función pública que aborda la ordenación, transformación, conservación y el control del uso del suelo, del vuelo y del subsuelo; su urbanización y edificación; y la regulación del uso, de la conser-

\footnotetext{
${ }^{6}$ Ya sea por su valor de uso como fuente de recursos, soporte de actividades y receptor de residuos, ya sea por su valor de cambio a partir de la generación de las expectativas de obtención de rentas a partir de su urbanización o de la transformación de su uso urbano.

7 El planeamiento urbanístico o planificación urbana es el conjunto de instrumentos técnicos y normativos que se redactan para ordenar el uso del suelo y regular las condiciones para su transformación o, en su caso, conservación.
}

vación, y de la rehabilitación de las obras, los edificios y las instalaciones". Dicha función se articula básicamente a través de los instrumentos de planificación y gestión y de los mecanismos de disciplina urbanística.

En relación con esta función pública del urbanismo, desde la aprobación de la Ley 2/2002 de urbanismo, la normativa incluye entre los principios rectores de la actuación urbanística (junto al de desarrollo urbano sostenible, el del ejercicio del derecho a la propiedad, el reparto equitativo de cargas y beneficios, etc.) el de la publicidad y la participación en los procesos de planeamiento y gestión urbanística. Así, el artículo 8.1 del Ley 3/2012 señala que "se han de garantizar y fomentar los derechos de iniciativa, información y participación de los ciudadanos en los procesos urbanísticos de planificación y gestión"; del mismo modo, el artículo 15.2 del reglamento de la ley de urbanismo (D305/2006), apunta "las administraciones públicas han de fomentar la participación social en la actividad urbanística y en especial en la elaboración y tramitación de los instrumentos de planeamiento urbanístico".

En consecuencia, al margen de reforzar la regulación de las formas de acceso, contenidos y períodos de exposición relacionados con la información pública de los planes y proyectos ${ }^{8}$ (cuestión sobre la que no haremos énfasis al ser similar a la existente en otras Comunidades Autónomas), la normativa urbanística catalana reciente incorpora dos novedades fundamentales en relación con la participación ciudadana: la obligatoriedad de aprobar un programa de participación ciudadana, en el caso de la formulación o revisión de instrumentos de planificación general, y la posibilidad de que los ayuntamientos constituyan consejos

\footnotetext{
${ }^{8}$ Cuestión de significativo interés y en la que se sigue profundizando y generando mejoras desde su primera inclusión en la normativa urbanística estatal La ley 19/1975 de reforma de la ley sobre régimen del suelo y ordenación urbana.
} 
asesores urbanísticos. A continuación trataremos cada uno de estos aspectos.

\section{La Memoria de Participación de los instrumentos de planificación general}

Tal y como señalábamos con anterioridad, la normativa catalana (art. 59.3 de la Ley $3 / 2012$ y art. 105.2 D305/2006) exige la incorporación en la memoria de los Planes de Ordenación Urbanística Municipal (POUM) ${ }^{9}$ del programa de participación ciudadana que el ayuntamiento haya aplicado a lo largo del proceso de formulación y tramitación del plan. Dicha obligación se hace extensiva igualmente a los futuros Plan Director Urbanístico Metropolitano y del Plan de Ordenación Urbanística Metropolitano (tal y como determina a Ley $31 / 2010$, del 3 de agosto del Área Metropolitana de Barcelona). Concretamente, el reglamento de la ley de urbanismo (Decreto 305/2006) establece que dicho programa de participación:

- Ha de ser aprobado simultáneamente al acuerdo que determine el inicio de los trabajos de formulación y revisión de las figuras de planeamiento general (art. 105.2).

- Ha de ser incluido en la memoria descriptiva y justificativa de dicho plan, haciendo referencia tanto a la descripción de las medidas y acciones realizadas con objeto de informar y promover el debate, sintetizando las aportaciones y resultados derivados de las alegaciones y sugerencias presentadas durante el periodo de información pública, e identificando las modificaciones o aspectos incorporados al plan a partir de las aportaciones o resultados del programa de participación ciudadana (art. 69.2).

En cualquier caso, cabe destacar que en ningún momento la normativa especifica la obligatoriedad de utilizar metodologías concretas, ni se apunta la

9 Figura equivalente a los planes generales de ordenación urbana de otras comunidades autónomas. posibilidad de que las aportaciones ciudadanas sean de carácter vinculante en algún supuesto, ni se exige la elaboración y publicación de un documento de evaluación del proceso participativo desarrollado.

En la práctica, el grado de aplicación de la normativa ha sido avanzado, si bien cabe destacar que no en todos los casos se ha cumplido con los mínimos legales exigidos y que en la mayoría de ellos no es posible conocer el grado de relación entre las aportaciones ciudadanas realizadas y las propuestas.

Así, de acuerdo con los datos que con fecha de diciembre de 2009 aportaba el informe La participació ciudadana en l'urbanisme: els Plans d'Ordenació Urbanística Municipal (Martí-Costa et al., 2010) a partir de los datos de los 93 municipios que habían aprobado un plan general entre 2003 y 2009 cabe destacar que si bien en el $92 \%$ de los casos era posible el acceso telemático a la memoria del POUM a través del Registro de Planeamiento Urbanístico de la Generalitat de Catalunya ${ }^{10}$, únicamente en un $75 \%$ se incluía el programa de participación ciudadana en la memoria del POUM, y sólo en el $49 \%$ de ellos se publicaba íntegramente en el Boletín Oficial de la Provincia correspondiente, tal y como determina el marco legal vigente. Del mismo modo, únicamente en el $23,1 \%$ de los casos el programa de participación incluido en la memoria recoge las aportaciones ciudadanas realizadas.

Por lo que se refiere al contenido metodológico, de los programas de participación de POUMs publicados en el BOP íntegramente, un 83,9\% incorporaba la realización de talleres, un 59,1\% la realización de actividades informativas, y un $37,8 \%$ contaba con algún mecanismo de participación individual.

\footnotetext{
10 En el $62,2 \%$ de los casos dicha memoria era también accesible a través de la web municipal.
} 


\section{Los Consejos Asesores Urbanísticos}

La otra gran novedad del marco normativo catalán en relación con la participación ciudadana se identifica, como decíamos con anterioridad, con la posibilidad de que los ayuntamientos constituyan consejos asesores urbanísticos (art. 8.2 de la Ley 3/2012).

Tal y como señala el reglamento de la ley (art. 24 del D/305/2006), dichos órganos (integrados por representantes de administraciones públicas, corporaciones, asociaciones e instituciones de la sociedad civil, expertos y ciudadanos a título individual) tienen un carácter participativo, informativo y deliberativo, y pueden proponer medidas y actuaciones dentro del programa de participación ciudadana, formular criterios y alternativas de ordenación, considerar las propuestas para garantizar los objetivos de desarrollo sostenible, opinar sobre las alegaciones presentadas, y estudiar las propuestas y medidas para fomentar la participación durante la tramitación de las figuras del planeamiento.

En la práctica, su grado de implantación ha sido bastante limitado. Así, a finales de 2009 (ob. cit.), únicamente un $15 \%$ de los municipios que habían iniciado la tramitación de la formulación o revisión de su planeamiento general habían constituido Consejos Asesores Urbanísticos; de estos, en un $88 \%$ de los casos no se había abierto su composición a la participación individual de los ciudadanos. Del mismo modo, la vida de dichos Consejos Asesores Urbanísticos se ha limitado a la propia elaboración del plan, desde el inicio de los trabajos hasta su aprobación definitiva.

\section{La participación ciudadana en los Proyectos de Intervención Integral de la “Ley de barrios"}

La regeneración urbana integral ${ }^{11}$ constituye una

11 Una posible definición es "Conjunto de intervenciones que
conjugan la rehabilitación física sobre edificios, incluyendo su
eventual renovación (demolición y construcción de nuevos práctica heterogénea, no consolidada pero en progresivo fortalecimiento, y meramente instrumental que se define de manera diversa en cada territorio en función de su organización políticocompetencial, de las características del sector privado, del tercer sector, de las necesidades del territorio, y de la perspectiva teórica e ideológica de los equipos de gobierno (Van der Bergh, 2004; Álvarez y Roch, 2010; Aparicio y Di Nanni, 2012; Verdaguer y Velázquez, 2012).

Una de las aproximaciones más habituales a la materia descansa en la consideración de las iniciativas de regeneración urbana integral como instrumentos dirigidos a mitigar las consecuencias de los procesos de segregación socioespacial generados por la diferente capacidad de elección del lugar y el tipo de residencia de los grupos de población de acuerdo con la renta de que disponen (Lefebvre, 1969 y 1974; Capel, 1975; Harvey, 1977, 2001; Smith, 2008; Nel.Lo, 2008; Llop et al., 2008; Martí-Costa et. al., 2009, Mongil, 2010),

Es en este contexto en el que las iniciativas de regeneración urbana integral han alcanzado en Cataluña un importante grado de desarrollo y calado institucional y social desde la aprobación de Ley 2/2004, de 4 de junio, de mejora de barrios, áreas urbanas y villas que requieren de una atención especial ${ }^{12}$, conocida popularmente como “Ley de Barrios"13.

edificios), con la intervención sobre el especio público o sobre los equipamientos, y con actuaciones de carácter social, económico o ambiental sobre una zona urbana en la que confluyen condiciones urbanas, sociales y económicas marcadamente desfavorables con respecto a las condiciones medias en las zonas urbanas del conjunto del país (Aparicio y $\mathrm{Di}$ Nanni, 2012).

12 Primer marco legal aprobado en el estado español dirigido específicamente a la mejora de barrios con problemas y a la lucha contra los procesos de segregación socioespacial (desarrollado reglamentariamente por el Decreto 369/2004, de 7 de septiembre).

13 En todo caso, las iniciativas de regeneración urbana integral ya habían aparecido en Cataluña anteriormente, por ejemplo a través de la Iniciativa Comunitaria URBAN (después URBANA), si bien no habían tenido ni tienen el calado institucional, territorial, económico y social de la "Ley de Barrios", razón por la que no hemos procedido a analizarlas a lo largo 
Dicha ley no cabe entenderla como el marco que regula los derechos y deberes de los ciudadanos de un barrio determinado, sino que básicamente constituye un instrumento que, reconociendo la existencia de procesos de segregación socioespacial, determina la intervención en áreas que requieren de atención especial. En la práctica, la "Ley de Barrios" se configura como un fondo financiero para financiar proyectos de intervención integral de iniciativa y ejecución municipal destinados a la mejora de aquellos barrios que por sus características requieran de una atención especial por parte de la Administración.

Con objeto de instrumentalizar el proceso, la Generalitat de Cataluña abre una convocatoria de ayudas (entre 2004 y 2010 con carácter anual, si bien el contexto de crisis económica y financiera ha hecho que no se hayan realizado nuevas convocatorias desde la fecha) con un período concreto para que los ayuntamientos que así lo deseen entreguen propuestas de Proyectos de Intervención Integral que desean que sean cofinancia$\operatorname{dos}^{14}$ por la Generalitat. Entre las características que han de cumplir los proyectos para ser financiados por la Generalitat destaca el hecho de que han de dirigirse a áreas donde se combinan problemas de involución urbanística, pérdida o crecimiento demasiado acelerado de población y déficit económicos y sociales (Núcleos antiguos, Polígonos de vivienda, y/o Áreas de urbanización marginal), y deben plantear una estrategia y un programa de actuaciones integral que prevea actuaciones en los siguientes campos:

- Mejora del espacio público y dotación de espacios verdes.

del presente artículo.

14 En principio un $50 \%$ del proyecto era financiado por la Generalitat de Cataluña y el 50\% restante entre el ayuntamiento y otras fuentes de financiación (principalmente particulares, sobre todo en actuaciones de rehabilitación), con un límite máximo de inversión total de más de 20 millones de $€$. No obstante desde el año 2009 se ha abierto una doble convocatoria, en la que en el caso de municipios menores de 10.000 habitantes el porcentaje de cofinanción de la Generali-
- Rehabilitación y equipamiento de los elementos colectivos de los edificios.

- La provisión de equipamientos para uso colectivo.

- La incorporación de tecnologías de la información de los edificios.

- El fomento de la eficiencia energética, el ahorro en el consumo de agua y el reciclaje de residuos.

- La equidad de género en el uso del espacio urbano y los equipamientos.

- La accesibilidad y la supresión de barreras arquitectónicas.

- El desarrollo de programas que comporten una mejora social, urbanística y económica del barrio.

Entre 2004 y 2010 se realizaron siete convocatorias, que han dado lugar a la financiación y puesta en marcha de 141 Proyectos de Intervención Integral, y que han significado un compromiso de inversión pública global cercano a los 1.400 millones de euros ${ }^{15}$.

Sobre la base de la importancia del refuerzo del capital social, de empoderamiento y corresponsabilización de las comunidades en los proyectos, la propia "Ley de Barrios" y el reglamento que la desarrollan incorporan la participación ciudadana como un elemento de valor.

Así, el artículo 12 del Decreto 369/2004 que despliega la ley ("Prioridad en la financiación y criterios de evaluación de los proyectos") establece que de los 114 puntos en juego para valorar la calidad de los proyectos, un máximo de 10 tiene que ver con los valores cualitativos del proyecto presentado, entre los que se tienen en cuenta las características generales del proyecto en relación

\footnotetext{
tat puede llegar a cubrir el $75 \%$ de la inversión total.

15 En 2009, El reparto de la inversión comprometida por campo de actuación era el siguiente: Espacio público: 46,7\%; Elementos colectivos edificios: 9,5\%; Equipamientos: $22,4 \%$; Tecnologías en edificios: 1,0\%; Sostenibilidad desarrollo urbano: 3,9\%; Equidad de género: 2,1\%; Accesibilidad: $6,6 \%$; Mejora social y económica del barrio: 8,0\% (Generalitat de
} 
con identificación de los problemas existentes, la coherencia y adecuación de las estrategias para corregir los problemas detectados, la concreción de los objetivos, la justificación de la viabilidad de los proyectos, el grado de participación ciudadana y la valoración del impacto del proyecto en el ámbito de actuación.

Por lo que se refiere al papel de la participación ciudadana en la evaluación y seguimiento de los proyectos, la ley es más precisa. Así, los artículos 12 y 18 del reglamento que desarrolla la ley (D369/2004), exigen la creación de un Comité de Evaluación y Seguimiento integrado por representantes de la Generalitat de Catalunya y del ayuntamiento, así como por entidades vecinales, agentes económicos y sociales y asociaciones ciudadanas. Dicho Comité, cuyas funciones son las de establecer los mecanismos de coordinación e información, fomentar la participación ciudadana, realizar el seguimiento de las actuaciones, y elaborar y elevar a la Comisión de gestión del Fondo de fomento del programa de barrios de la Generalitat de Catalunya el informe de evaluación final del proyecto, ha de reunirse un mínimo de tres veces al año.

En la práctica, la incorporación de iniciativas participativas en la elaboración y desarrollo de los proyectos ha sido elevada, y progresivamente creciente aunque variable en función de la valoración positiva que se realizaba desde el fondo de barrios (que concede las ayudas económicas a los proyectos) de la incorporación de iniciativas participativas, de la voluntad municipal y de las demandas sociales.

Así, si bien es imposible tener datos rigurosos sobre cuántos de los proyectos presentados a las diferentes convocatorias incorporaban en las propuestas la realización de procesos de participación ciudadana, situación condicionada en gran medida por el reducido plazo dado entre el mo- mento de aprobación de la convocatoria y el de la presentación de las solicitudes de proyectos a financiar (dicho periodo ha oscilado entre el mes y medio y los tres meses), la mayoría de los proyectos han realizado sesiones y espacios participativos (principalmente en forma de talleres y/o audiencias) para conocer e incorporar la opinión, conocimiento y demanda ciudadana en los proyectos. A modo de ejemplo, cabe destacar que los 55 proyectos (41 de los cuales han resultado beneficiarios del fondo de barrios) elaborados con apoyo de la Diputación de Barcelona) incorporaban en los análisis, estrategias y actuaciones los resultados de los procesos participativos desarrollados en cada uno de los proyectos.

Del mismo modo, tal y como muestran los datos que hemos extraído a partir del análisis del Atlas de Proyectos de Intervención Integral de la Generalitat de Cataluña ${ }^{16}$, sí que podemos afirmar que la participación ciudadana juega un papel fundamental en la ejecución y desarrollo de los 141 proyectos beneficiarios del fondo de barrios entre 2004 y 2010 . En este sentido:

- Un $60,3 \%$ de los proyectos incorporan entre sus actuaciones iniciativas participativas, la mayoría bajo la forma de programas o planes de participación ciudadana ligados al propio proyecto $(68,2 \%$ de los casos), y en otras ocasiones asociadas e interrelacionadas a actuaciones de información y comunicación. La incorporación de acciones participativas no presenta variaciones notables en función del tamaño del municipio o del tipo de barrio. En todo caso, cabe destacar su progresivo fortalecimiento a lo largo del tiempo (así mientras que en 2004 y 2005 únicamente un $30,80 \%$ y un $23,50 \%$ de los proyectos incorporaban actuaciones ligadas a la

\footnotetext{
16 Dónde se recogen de manera georreferenciada todas las actuaciones (descripción y presupuesto incluido) de todos los proyectos de intervención integral beneficiarios del fondo de barrios: http://www6.gencat.cat/atlasbc/.
} 
participación, desde 2007 el porcentaje no ha sido nunca inferior al $68 \%$ ).

- El presupuesto total de las actuaciones de participación ciudadana incluidas en los proyectos asciende a la significativa cantidad de 9.243.492 de euros, si bien existen diferencias notables en los volúmenes de inversión en participación ciudadana entre los proyectos. Así, un $14,18 \%$ de los proyectos que incorporan acciones participativas destinan a las mismas más de 300.000 euros, pero un $22,69 \%$ de los mismos destina menos de 30.000 euros). Dichas variaciones guardan relación en muchos casos con el tamaño de los municipios donde se localicen los proyectos y con el presupuesto global de los mismos.

- Por lo que se refiere al contenido de las actuaciones participativas, si bien ha sido imposible realizar una aproximación exhaustiva y sistemática, nuestra experiencia sobre la base de los proyectos a los que se presta apoyo desde la Diputación de Barcelona y de otros muchos de los que se tiene conocimiento directo, es que las actuaciones participativas se han articulado básicamente en la realización de procesos ligados a procesos de mejora del espacio público y de programación de usos de equipamientos, a acciones de fomento del asociacionismo, y a la concreción de los mecanismos y formas de evaluación y seguimiento del proyecto.

Precisamente, sobre esta cuestión, y en relación con los Comités de Evaluación y Seguimiento cabe destacar que, tal y como exige la ley, todos los proyectos han constituido sus comités, los cuales se reúnen periódicamente. No obstante, no se disponen de datos sistemáticos para poder profundizar en la composición de los mismos, la periodicidad de sus encuentros, o el grado de cumplimento de las funciones que tienen legalmente asignadas.

\section{Valoración y propuestas para una mejora cualitativa del urbanismo participativo en Cataluña}

Tal y como se ha visto en los apartados anteriores, a lo largo de los últimos años se ha producido un refuerzo de la participación ciudadana como política pública que, en Cataluña y en relación con el urbanismo, se ha manifestado de forma especialmente significativa en relación con el desarrollo de acciones participativas relacionadas con los instrumentos de planificación urbanística y de regeneración urbana integral. La valoración de los avances y límites de este urbanismo participativo en Cataluña puede sintetizarse en tres grandes reflexiones:

- El significativo, aunque mejorable, avance en la incorporación de la participación ciudadana a la normativa que regula los instrumentos de planificación urbanística y de rehabilitación urbana integrada.

- La progresiva, aunque incipiente, evolución de los modelos de participación ciudadana aplicados a los iniciativas urbanísticas.

- La limitada influencia de los procesos participativos en los procesos de elaboración y de toma de decisiones de los instrumentos de planificación urbanística y de regeneración urbana integral.

A continuación hablaremos de cada una de estas cuestiones.

\section{El significativo, aunque mejorable, avance en la incorporación de la participación ciudadana a la normativa que regula los instrumentos de planificación urbanística y de rehabilitación urbana integrada}

Tal y como se ha mostrado en el capítulo anterior, tanto la normativa urbanística como la de intervención integral en áreas urbanas, se han hecho 
eco del principio rector del Estatuto de Autonomía de Cataluña referente al Fomento de la Participación.

En este sentido, la normativa urbanística ha reforzado y mejorado cualitativamente los procesos de información pública, y ha incorporado en los últimos años la obligatoriedad de aprobar un programa de participación ciudadana en la formulación o revisión de instrumentos de planificación general, así como la posibilidad de que los ayuntamientos constituyan consejos asesores urbanísticos.

Ambas cuestiones se han ido reforzando a lo largo del tiempo y se han mantenido a lo largo de los sucesivos cambios en la normativa urbanística, hasta la actual Ley 3/2012 del 22 de febrero, de modificación del texto refundido de la Ley de Urbanismo (que de hecho ha supuesto significativas modificaciones en otras cuestiones relacionadas con el urbanismo). En todo caso, aún está pendiente la adaptación a la ley 2/2012 del Reglamento de la Ley de Urbanismo que deberá aprobarse antes del verano de 2013 (actualmente sigue vigente el reglamento aprobado por el Decreto $305 / 2006$ ), por lo que, dado que las determinaciones concretas sobre el programa de participación se regulan por reglamento, aún podrían producirse cambios significativos (en todo caso no se han tenido noticias hasta la fecha de que estos vayan a producirse, ni existe un debate visible y generalizado sobre la necesidad o posibilidad de cambiar las determinaciones referidas a la participación ciudadana en la elaboración de los instrumentos de participación).

Por su parte, la normativa sobre intervención integral en áreas urbanas ha incorporado la participación ciudadana como un elemento intrínseco a los propios proyectos que se valora positivamente para la financiación de los mismos por parte de la Generalitat de Catalunya y que ha de estar incorporada en los mecanismos de evaluación y se- guimiento de los mismos.

Por lo tanto, podríamos afirmar que en el caso catalán se ha satisfecho al menos en parte, una de las principales demandas que se realizaban (tanto por parte de los expertos como de los ciudadanos) en relación con la materia, como es el reconocimiento legal de la incorporación de la participación ciudadana en la práctica urbanística. No obstante, y al margen del significativo avance producido, a nuestro juicio cabría la posibilidad de reforzar y mejorar cualitativamente esta incorporación legal de la participación ciudadana:

- En relación con los instrumentos de planificación urbanística, dado que en la práctica, el planeamiento general sufre significativas modificaciones antes de su ejecución con objeto de adaptarlo a los cambios de contexto económico, social, o político que se van produciendo, cabría la posibilidad de que la normativa fuese un poco más allá, y extendiera la obligatoriedad de realizar programas de participación en relación con algunas de las modificaciones puntuales.

Dicha cuestión adquiere una significativa importancia en el caso del ámbito metropolitano barcelonés, regulado por el Plan General Metropolitano desde el año 1976, y en el que las significativas transformaciones urbanísticas (en muchos casos lógicas) que se producen no están obligadas a incorporar un proceso de participación ciudadana más allá del proceso de información pública, excepción hecha del ayuntamiento de Barcelona ${ }^{17}$.

Al efecto, paralelamente a lo ya regulado en el caso de la obligación de realizar un nuevo

\footnotetext{
17 En este sentido, cabe destacar que el artículo 70 de la Ley 22/1998, de 30 de diciembre, de la Carta municipal de Barcelona, señala que en la elaboración de los instrumentos de ordenación y de los proyectos de urbanización y de obras se ha de fomentar la participación ciudadana, y apunta que el Reglamento del Consejo Municipal deberá establecer los supuestos en los que será obligatorio, además del trámite de información pública, el seguimiento de otros procedimientos de participación ciudadana.
} 
período de información pública (art.112 del D305/2006), podría exigirse la realización de procesos o espacios de participación ciudadana en la formulación de modificaciones puntuales de plan general cuando puedan generar cambios substanciales relacionados con la adopción de nuevos criterios respecto a la clasificación del suelo, la estructura general y el modelo de ordenación del territorio. Del mismo modo podría determinarse al menos la recomendación de la realización de procesos o espacios de participación ciudadana cuando dichas modificaciones puedan producir cambios en la clasificación del suelo, en las previsiones sobre sistemas urbanísticos generales, y en las cualificaciones que generen un incremento del aprovechamiento urbanístico.

Igualmente cabría garantizar el cumplimiento de todas las determinaciones legales existentes en relación con los programas de participación ciudadana que se realizan, sobre todo en relación con su publicación integra en el Boletín Oficial de la Provincia y la identificación en la memoria de las aportaciones ciudadanas realizadas y de cuales de ellas se han incorporado finalmente en el plan general ${ }^{18}$.

- En relación con los instrumentos de regeneración urbana integral, dado el valor de la participación ciudadana para el refuerzo de la cohesión social y la maximización del capital social y ambiental del territorio, cabría la posibilidad de que la normativa exigiera, aunque en la mayoría de los proyectos los ayuntamientos ya la incluyan voluntariamente,

\footnotetext{
18 Recordamos que en diciembre de 2009 únicamente el 49\% de los Planes de Ordenación Urbana Municipal aprobados desde 2002 habían publicado íntegramente la memoria de participación, y que únicamente el $23,1 \%$ hacían referencia concreta a las aportaciones ciudadanas realizadas e incorporadas (Martí-Costa et. Alt., 2010).
}

que los proyectos que solicitan ser objeto de subvención incluyan una memoria de participación ${ }^{19}$. Del mismo modo, de cara a futuras convocatorias de ayudas para la ejecución de proyectos de intervención integral (desde el año 2010, dado el actual contexto de crisis y las limitaciones económicas de la Generalitat de Catalunya, no se han abierto nuevas convocatorias) sería recomendable revalorizar la puntuación, visualización e influencia que en la decisión sobre los proyectos a financiar por la Generalitat de Catalunya tiene la participación ciudadana realizada en la definición del proyecto 0 prevista en su ejecución.

\section{La progresiva, aunque incipiente, evolución de los modelos de participación aplicados a las iniciativas urbanísticas}

En relación con la manera en la que se ha aplicado la participación ciudadana en los instrumentos de planificación urbanística y regeneración urbana integral, tal y como se ha podido intuir a partir de los datos mostrados en el segundo capítulo, podemos afirmar que se ha asistido a una evolución y maduración de los modelos de urbanismo participativo. En este sentido, se han sentado las bases para configurar un modelo de participación progresivamente deliberativo que vaya más allá de la simple información o consulta a la ciudadanía.

En este sentido, si tomamos en consideración con la categorización de modelos de participación en el urbanismo elaborado por Martínez en 2011, podríamos afirmar que estamos asistiendo a una importante evolución desde modelos unidimensionales (puramente tecnocrático, o de cooperación entre expertos y técnicos mediante la cual

\footnotetext{
19 Con contenidos y funciones similares a las que tiene la memoria de participación ligada a los planes de ordenación urbana municipal.
} 
éstos ayudan a "traducir" las demandas, necesidades e inquietudes de aquellos) a modelos cada vez en mayor proporción multidimensionales que ponen el acento en la importancia del intercambio, el diálogo, y el aprendizaje mutuo a través del diálogo y acción conjunta y la posibilidad de establecer consensos.

No obstante, si tomamos como referencia la definición de modelos de participación aplicados a instrumentos urbanísticos elaborada por Glass en $1979^{20}$, podríamos apuntar que si bien en la definición y ejecución de los proyectos de intervención integral de la ley de barrios es generalizada la configuración de modelos deliberativos que incluyen acciones y procesos de información/ consulta, mecanismos de apoyo a la decisión (órganos consultivos o asesores, entrevistas, encuestas, etc.) y espacios de diálogo y deliberación ciudadana (talleres, etc.), en el caso de los procesos participativos ligados al planeamiento urbanístico ésta es aún incipiente y en la mayoría de los casos las acciones se han ceñido a la información y consulta. Así, en diciembre de 2009 únicamente el $16 \%$ de los acciones participativas realizadas en los procesos de elaboración o revisión de Planes Generales de Ordenación Urbana se correspondía con un "modelo deliberativo" (Martí-Costa et. al., 2010).

De cara al futuro, los principales retos en relación con los modelos de aplicación de la participación ciudadana en el urbanismo catalán pasan por el refuerzo de fórmulas que garanticen una deliberación de calidad, y progresen a la codecisión de algunas intervenciones, casuística en la que, lógicamente se ha avanzado más en relación con iniciativas ligadas a la mejora del espacio público, que en las relacionadas con los instrumentos de

\footnotetext{
20 Seguida entre otros por Martí-Costa y otros en 2010 en el análisis de la aplicación de la participación ciudadana en los Planes Generales.
}

planificación urbanística ${ }^{21}$.

Del mismo modo, cabe reforzar y mejorar significativamente la relación entre las aportaciones ciudadanas y las propuestas finales, con objeto de garantizar que los procesos participativos van más allá del mero cumplimiento de la normativa vigente o de la mera voluntad de dotar de mayor transparencia y visibilidad a la acción pública.

En todo caso, la mayor o menor calidad de la participación ciudadana ligada al urbanismo no descansa tan sólo en la naturaleza del modelo participativo aplicado, sino también, y de forma destacada, en la interacción del mismo con la naturaleza de la práctica urbanística y la forma en la que se han configurado tradicionalmente los procesos de elaboración y la toma de decisiones.

A continuación abordamos está cuestión.

\section{La limitada influencia de los procesos participativos en los procesos de elaboración y de toma de decisiones de los instrumentos de planificación urbanística y de regeneración urbana integral}

La consulta ${ }^{22}$ de la documentación de los instrumentos de planificación urbanística (de obligada accesibilidad en el Registro de Planeamiento Urbanístico de la Generalitat de Catalunya) y de los proyectos de intervención integral de la ley de barrios (accesibles de forma resumida y parcial en el Atlas de Proyectos de Intervención Integral de la Generalitat de Cataluña) y la experiencia profesional de la práctica urbanística de estos años, nos permite apuntar que el impacto que la participación ciudadana ha tenido sobre el modelo

\footnotetext{
${ }^{21}$ Accesibles en

http://ptop.gencat.net/rpucportal/AppJava/cercaExpedient.do?r eqCode=load y en http://www6.gencat.cat/atlasbc/ respectivamente.

22 Según el trabajo de Font y Galais citado en la bibliografía y reseñado a lo largo del segundo apartado de este artículo, en 2009 hasta un $20 \%$ de las iniciativas participativas relacionadas con la ordenación urbana habían estado relacionadas con el codiseño de la intervención, en la mayoría de los casos, como decimos, ligadas a la mejora del espacio público.
} 
de definición, gestión y toma decisiones de la práctica urbanística ha sido hasta la fecha poco significativo, y que, cómo ya apuntaban algunos expertos (Friedmann, 1991), a través de la deliberación e interacción entre los ciudadanos y las estructuras político-técnicas de la administración no es fácil que se produzcan variaciones estructurales en la manera de ejecutar las políticas públicas.

De hecho, en una primera aproximación, el análisis y la consulta de los instrumentos urbanísticos resultantes no permiten atisbar cambios sustanciales en las propuestas, programas y modelos. En este sentido, la evolución hacia una mayor consideración no ya sólo de la participación ciudadana, sino de la integralidad y del fortalecimiento de la dimensión socioambiental del urbanismo, sobre todo en relación con la planificación urbanística se aprecia con más facilidad en las memorias y documentos de diagnosis (es decir en la dimensión simbólico-discursiva de los instrumentos) que en los programas y propuestas de ordenación. Incluso cuando los instrumentos incorporan memorias de los procesos de participación realizados, en muchas de las ocasiones no se identifica o no es fácilmente contrastable la influencia, incidencia y valor de las aportaciones ciudadanas en relación con las propuestas finales que se realizan.

En este sentido, la práctica urbanística sigue girando en torno a una "manera de hacer" que pese a su indudable y ya comentada función social, adolece de seguir dominada por un supuesto rigor técnico-funcional que fundamenta unas decisiones que en realidad no ejerce (ni tiene porqué ejercer), de no favorecer la visibilidad de los mapas de agentes que tienen verdadera influencia sobre el proceso, de estar excesivamente subordinada a los intereses e iniciativas privadas tanto para su definición como su ejecución ${ }^{23}$, y de estar condicionada al hecho de ser una de las principales vías de financiación de las exiguas arcas municipales (Lefebvre, 1968 y 1974; Capel, 1975; Ezquiaga,1998; Saravia, 1998; Terán, 1999 y 2009; Boira, 2003; Martínez, 2006; Delgado, 2007; Naredo et. al., 2008).

Por su parte, la regeneración urbana integral continúa teniendo un carácter eminentemente instrumental y financiero. Por ello, la naturaleza y características de los proyectos se ven excesivamente subordinados a las condiciones específicas de las bases de financiación de las diferentes convocatorias y programas. Además, dado el carácter embrionario del enfoque, las inercias y características de las estructuras administrativas preexistentes, y los apretados calendarios de ejecución y justificación de la financiación de los proyectos, presentan en muchas ocasiones dificultades para adquirir un verdadero carácter integral que vaya más allá de la yuxtaposición de actuaciones sectoriales diversas (Martí-Costa y Parés 2009; Álvarez y Roch, 2010; Aparicio y Di Nanni, 2012).

En consecuencia se corre el riesgo de que en muchos casos los procesos de participación ligados al urbanismo se conviertan en procesos excesivamente teledirigidos por parte de la administración pública o que en el mejor de los casos se limiten simplemente a garantizar los procedimientos legalmente establecidos (Boira, 2003; Martínez, 2006; Delgado, 2007).

Por ello, la mejora de la calidad del urbanismo participativo, descansa, junto a su vinculación normativa o los modelos de participación que se apliquen, también en aspectos directamente relacionados con la especificidad de la práctica urba-

\footnotetext{
23 Situación en la que tiene mucho que ver la escasez de suelo propiedad de las administraciones públicas y los escasos recursos económicos de que éstas disponen para ejecutar las actuaciones necesarias para materializar el modelo urbano previsto.
} 
nística, como por ejemplo:

- La incorporación de la participación desde el mismo comienzo de la elaboración de los instrumentos con objeto de evitar su posible subordinación y dependencia a enfoques y propuestas ya maduradas desde el nivel político y técnico, y de facilitar la incorporación de las aportaciones ciudadanas ya en la primera propuesta que se elabore, sea pública o no (Velázquez y Verdaguer, 2011).

- La identificación y visualización de los agentes públicos y privados implicados e influyentes en la toma de decisiones, con objeto de hacer más transparente el proceso de tomas de decisiones y facilitar tanto la confianza de la ciudadanía como su percepción de la complejidad del proceso de toma de decisiones (ob. cit.).

- La necesidad de comunicar y hacer visible el carácter transversal y flexible del urbanismo. Por lo tanto de no limitar excesivamente la dinámica participativa al marco competencial, los límites de los instrumentos y la interpretación tecnocrática de los mismos (Martí-Costa y Parés, 2009; Mongil, 2011, Velázquez y Verdaguer, 2011) y de entender y hacer entender los planes y proyectos como un punto de apoyo, un marco inicial de discusión, siempre revisable, y una base para el proceso de coordinación de múltiples actores (Faludi, 2002 y 2007; Farinós y Romero, 2007). Estas cuestiones adquieren una especial importancia en al actual contexto de crisis económica y financiera que puede ayudar a reorientar y evolucionar la práctica tradicional del urbanismo.

- La abertura del proceso de definición de los límites y los temas a tratar en la dinámica participativa a todos los agentes implicados. De esta manera, sin perder la capacidad de concreción de los límites y reglas del juego del proceso por parte del ayuntamiento, se ayudaría a mitigar que la dinámica participativa sea excesivamente teledirigida por parte de la administración, sea fagocitada por los temas más conflictivos o por determinados intereses particulares, pase por alto la dimensión y repercusión económica de las intervenciones, o se ciña en exclusiva a cuestiones o principios más o menos generales de ambigua y difícil plasmación posterior en las normativas, propuestas de ordenación y programas de actuación resultantes (Saravia, 1998; Martínez 2006).

- La interpretación (más allá de la recogida sistémica y su incorporación o no en las propuestas) de las opiniones y valoraciones de cada uno de los agentes que han participado en la dinámica participativa, con objeto de facilitar el conocimiento de las necesidades, demandas e imaginarios de los implicados, lo cual puede ayudar a gestionar mejor la complejidad del hecho urbano (Mongil, 2011).

- La propia valoración de las dinámicas participativas por parte de los ciudadanos y agentes que en ellas han participado, con objeto de no limitar el debate exclusivamente a los expertos.

En definitiva, podemos afirmar que a lo largo de estos últimos años se han sentado las bases teóricas y prácticas para la configuración de un urbanismo más participativo. En este sentido, podemos afirmar que se ha producido un avance significativo. No obstante cabe profundizar tanto en la mejora cualitativa de la normativa que ha de dar mayor solidez al proceso como en los modelos de aplicación de la participación ciudadana, haciendo especial énfasis no ya tanto en su mejora metodológica o su alcance como en su interacción con la práctica e inercia tradicional del urbanismo.

Todo ello, sin menoscabar la función social que 
ha tenido el urbanismo, y entendiendo la participación ciudadana como una forma de gobernanza (y no tanto como una política pública más) dentro de los límites de la democracia representativa y por tanto supeditada a las voluntades y decisiones del sistema democrático. Es decir, considerando que la participación ciudadana puede contribuir a hacer un mejor urbanismo.

\section{Referencias}

ÁLVAREZ, Alfonso y ROCH, Fernando (dir.). Regeneración urbana integrada en Europa: documento de síntesis. Valladolid: Instituto Universitario de Urbanística de la Universidad de Valladolid (encargo del Ministerio de Vivienda), 2010, p.56

<http://www.fomento.gob.es/NR/rdonlyres/B8F44 D9E-47ED-44DE-8FEC-

47FFE2FC630B/111525/3_survey_on_integrate d.pdf>. (Consultado el 11 de enero de 2013).

APARICIO, Antonio y DI NANNI, Roberta. Modelos de gestión de la regeneración urbana. Madrid: Sepes (Entidad Estatal de Suelo), 2011, p.162

BENABENT, Manuel. El interés general en la filosofía política. Un concepto ético y normativo necesario para la planificación territorial. Boletín de la Asociación de Geógrafos Españoles, 2010, ํo 53, p. 121-146.

BOBBIO, Luigi (Ed.). A più voci: Amministrazioni pubbliche, imprese, associazioni e cittadini nei processi decisionali inclusivi. Roma: Edizioni Scientifiche Italiane, 2004, p.152

BOIRA, Josep Vicent. La participación ciudadana y el urbanismo: ¿radicalizar la democracia o democratizar el espacio? Ciudades, arquitectura y espacio urbano (Colección Mediterráneo Económico), 2003, no 3, p. 317-332.

BRUGUÉ, Joaquim, GOMÀ, Ricard y SUBIRATS, Joan. Gobierno y territorio: del Estado a las redes. In SUBIRATS, Joan (Coord.). Redes, terri- torios y gobierno. Nuevas respuestas locales a los retos de la globalización. Barcelona: Diputación de Barcelona, 2002, p. 299-311.

BRUGUÉ, Joaquim. Una administración que habla es una administración que piensa. In SUBIRATS, Joan et al. Participación Ciudadana... para una Administración deliberativa. Zaragoza: Gobierno de Aragón, 2009, p. 55-72.

CAPEL, Horacio, Capitalismo y morfología urbana en España. Barcelona: Libros de la Frontera, 1983 ( $1 \stackrel{\text { a }}{ }$ ed. Barcelona, 1975), p.143

DELGADO, Germán J. La participación ciudadana en el planeamiento urbanístico y el fenómeno de la teledirección: Análisis crítico sobre el proceso de formación de los planes urbanísticos en España y la incidencia de la opinión de los ciudadanos en su redacción, tramitación y gestión posterior. Scripta Nova, Revista Electrónica de Geografía y Ciencias Sociales, 2007, Vol. XI, no $245 . \quad<h t t p: / / w w w . u b . e d u / g e o c r i t / s n / s n-$ 24512.htm>. (Consultado el 11 de enero de 2013).

DELGADO, Manuel. Barcelona: urbanismo versus urbano. In DIPUTACIÓN DE BARCELONA, Ciudades en (re)Construcción: necesidades sociales, transformación y mejora de barrios. Barcelona: Diputación de Barcelona, 2007, p.149157.

ESTEBAN, Juli. L'ordenació urbanística: conceptes, eines i pràctiques. Barcelona: Diputació de Barcelona, 2007, p. 281

EZQUIAGA, José María. ¿Cambio de estilo o cambio de paradigma? Reflexiones sobre la crisis del planeamiento urbano, Revista URBAN, 1998, no 2, p. 7-33.

FALUDI, Andreas y WATERHOUT, Bas. The Making of the European Spatial Development Perspective: No Masterplan. Routledge, London: RTPI Library Series, 2002.

FALUDI, Andreas. El Método Abierto de Coordinación en planificación territorial a escala de 
Unión Europea. In FARINÓS, Joaquim y ROMERO, Joan (Coords.). Territorialidad y buen gobierno para el desarrollo sostenible. Nuevos principios y nuevas políticas en el espacio europeo. Valencia: Publicaciones de la Universidad de Valencia, 2007, p. 79-102.

FARINÓS, Joaquim. Nuevas formas de gobernanza para el desarrollo sostenible del espacio relacional. Ería, 2005, oㅡ 67, p. 219-235.

FARINÓS, Joaquim y ROMERO, Joan (Coords.). Territorialidad y buen gobierno para el desarrollo sostenible. Nuevos principios y nuevas políticas en el espacio europeo. Valencia: Publicaciones de la Universidad de Valencia, 2007, p. 279

FONT, Joan y BLANCO, Ismael. Experiencias de participación ciudadana. Polis, la ciudad participativa. Participar en los municipios: ¿Quién?, ¿Cómo? Y ¿Por qué? Barcelona: Diputació de Barcelona, 2006.

FONT, Joan y GALAIS, Carolina. Experiències de democràcia participativa a Catalunya: un mapa analític. Barcelona: Generalitat de Catalunya, 2009.

FRIEDMANN, John. Planificación en el ámbito público. Madrid: INAP-MAP, 1991, p. 470

IGLESIAS, Felipe (Coord.). Urbanismo y democracia. Alternativas para evitar la corrupción. Madrid: Fundación Alternativas, 2007, p. 165

GENERALITAT DE CATALUNYA. Memoria de les subvencions a ens locals per promoure la participació ciudadana 2005-2010. (Documento de Trabajo). Barcelona: Generalitat de Catalunya, 2.010 .

<http://www20.gencat.cat/docs/governacio/Rela cions\%20Institucionals/03_Qualitat_democratica /03_Subvencions/02_M\%C3\%B3n\%20local/01_ Fo-

ment\%20de\%20la\%20participaciC3\%B3/memor ia.pdf>. (Consultado el 11 de enero de 2013).

GIDDENS, Anthony. La constitución de la sociedad: bases para la teoría de la estructuración.
Buenos Aires: Ed. Amorrortu, 1984, p. 412

GLASS, James J. Citizen Participation in Planning: The relationship Between Objectives and Techniques. Journal of the American Planning Association, 1979, vol 45, no 2, p. 180-189.

HABERMAS, Jürgen. The theory of Comunicative Action. Vol. 1: Reason and the Rationalisation of Society. London: Polity Press, 1984.

HEALEY, Paul. Collaborative Planning. Shaping places in Fragmented Societies. London: McMillan Press, 1997.

HARVEY, David. Urbanismo y desigualdad social. Madrid: Siglo XX Editores, 1977, p. 340

HARVEY, David. Espacios de capital: hacia una geografía crítica. Madrid: Aikal, Cuestiones de Antagonismo, 2001, p. 445

LEFEBVRE, Henri. El derecho a la ciudad. Barcelona: Ed. Península, 1969, p.169 (Primera edición en francés: 1968).

LEFEBVRE, Henri. La Production de l'espace. Paris: Anthropos, 1974, p. 485

Llop, Josep Maria, VALLS, Xavier, ALBORS, Judit y MONGIL, David. Ciudades en (re)construcción: ¿hacia una nueva cultura urbanística? In DIPUTACIÓN DE BARCELONA, Ciudades en (re)Construcción: necesidades sociales, transformación y mejora de barrios. Barcelona: Diputación de Barcelona, 2007, pp 9-25.

LÓPEZ, Fernando, BÁGUENA, José Antonio y MONGIL, David. Territorio y Participación Ciudadana. Cuaderno no 1. Zaragoza: Gobierno de Aragón, 2010, p. 30

MARCHIONI, Marco. Comunidad, participación y desarrollo: teoría y metodología de la intervención comunitaria. Madrid: Editorial Popular, 1999, p.187

MARTÍ, Joel, PASCUAL, Jordi, REBOLLO, Óscar (Coord.). Participación y desarrollo comunitario en medio urbano: experiencias y reflexiones. Madrid: lepala Editorial / Cimas, 2005, p. 307 MARTí-COSTA, Marc y PARÉS, Marc (Coord.). 
Llei de Barris: cap a una política de regeneració urbana participada i integral. Barcelona: Generalitat de Catalunya, 2009, p. 163

MARTÍ-COSTA, Marc (coord.). La participació ciudadana en l'urbanisme: els Plans d'Ordenació Urbanística Municipal: Informe Final. Barcelona: Àrea de Participació i moviments socials, Institut de Govern i Polítiques Públiques (encargo de la Generalitat de Catalunya), 2010. <http://www20.gencat.cat/docs/governacio/Quali tat\%20democr\%C3\%A0tica/02_Divulgacio,\%20f ormcio\%20\%\%20recerca/04_Recerca/Arxius/pla_ ordenacio_municipal.pdf>. (Consultado el 11 de enero de 2013).

MARTÍNEZ, Miguel. La participación social en el urbanismo, en los límites de la realidad. Boletín Ciudades para un mundo más sostenible, 2006, ำ 34 .

<http://habitat.aq.upm.es/boletin/n34/ammar.html

$>$ Consultado el 30 de mayo de 2012.

MARTÍNEZ, Miguel. Dimensiones múltiples de la participación ciudadana en la planificación espacial. Revista Reis - Revista Española de Investigaciones Sociológicas, 2011, no 13, p. 2142.

MONGIL, David. Intervención integral en barrios: conceptos, instrumentos y elementos de mejora. Revista Ciudades, 2010, oㅜ 13, p. 139-161.

MONGIL, David. Urbanisme i Participació. Revista "Territori i Ciutat", 2011, no 46. <http://www.diba.es/c/document_library/get_file? uuid $=4401625 b-6 f 26-49 b 9-9 c f 9-$

cc7a0c68cdc4\&groupld=1295730>. (Consultado el 11 de enero de 2013).

NAREDO, José Manuel, CARPINTERO, Óscar y MARCOS, Carmen. Patrimonio Inmobiliario $y$ balance de la economía española 1.995-2.007. Madrid: Fundación de las Cajas de Ahorros, 2008, p. 243

NEL.LO, Oriol. Contra la segregación urbana y por la cohesión social: la Ley de Barrios de Ca- taluña. In DIPUTACIÓN DE BARCELONA, Ciudades en (re)Construcción: necesidades sociales, transformación y mejora de barrios. Barcelona: Diputación de Barcelona, 2007, p. 233253.

PINDADO, Fernando. La participació ciutadana a la vida de les ciutats. Barcelona: Edicions Serbal, 1999, p. 193

PINDADO, Fernando. La participación ciudadana es la vida de las ciudades In SUBIRATS, Joan et al. Participación Ciudadana... para una Administración deliberativa. Zaragoza: Gobierno de Aragón, 2009, p.119-148.

ROMERO, Joan. La cooperación territorial en España: tradición, transición y nueva etapa. In VV.AA. Una Nueva Cultura del Territorio. Criterios sociales y ambientales en las políticas y el gobierno del territorio. Barcelona: Diputación de Barcelona, 2006, p. 487-504.

SAMPEDRO, José Luis y TAIBO, Carlos. Sobre política, mercado y convivencia. Madrid: Ed. Catarata, 2006, p. 160

SARAVIA, Manuel. El planeamiento urbano, otra vez en crisis. In VV.AA. El malestar urbano en la gran ciudad. Madrid: Talasa, 1998, p. 91-111.

SMITH, Neil. Gentrificación generalizada: de la anomalía local a la regeneración urbana como estrategia global urbana In DIPUTACIÓN DE BARCELONA, Ciudades en (re)Construcción: necesidades sociales, transformación y mejora de barrios. Barcelona: Diputación de Barcelona, 2007, p. 27-46.

SUBIRATS, Joan. La gobernabilidad de las políticas territoriales. Formulación participativa y gestión concertada. In VV.AA. Una Nueva Cultura del Territorio. Criterios sociales y ambientales en las políticas y el gobierno del territorio. Barcelona: Diputación de Barcelona, 2006, p. 389408.

SUBIRATS, Joan. Los espejismos de la razón y los caminos de la participación. In SUBIRATS, 
Joan et al. Participación Ciudadana... para una Administración deliberativa. Zaragoza: Gobierno de Aragón, 2009, p. 7-18.

SUBIRATS, Joan. Otra sociedad, ¿otra política?: de "no nos representan" a la democracia de lo común. Barcelona: Icaria, 2011, p. 104

TAIBO, Carlos. Nada será como antes. Sobre el movimiento 15-M. Madrid: Ed. Catarata, 2011, p. 86

TERÁN, Fernando de. Historia del urbanismo en España. Vol. III (siglos XIX-XX). Madrid: Cátedra, 1999, p. 397

TERÁN, Fernando de. El pasado activo: del uso interesado de la historia para el entendimiento y la construcción de la ciudad. Madrid: Akal / Textos de Arquitectura, 2009, p. 333

VAN DEN BERG, Leo, VAN DER MEER, Jan y BRAUN, Erik. National Urban Policies in the European Union. Rotterdam: EURICUR, 2004.

VELÁZQUEZ, Isabela y VERDAGUER, Carlos (Coord. Diputació de Barcelona). Urbanisme i participació: Iniciatives i reptes de futur: Conclusions del grup de treball sobre urbanisme i participació ciutadana en l'àmbit local. Barcelona: Diputació de Barcelona, 2011.

VELÁZQUEZ, Isabela y VERDAGUER, Carlos. Regeneración urbana integral: tres experiencias europeas innovadoras: Ille de Nantes, Coin Street y Barrio de La Mina. Madrid: Entidad Estatal de Suelo, 2011, p. 138

VILLASANTE, Tomás R. Las democracias participativas. De la participación ciudadana a las alternativas de la sociedad. Madrid: HOAC, 1995, p. 420

ZOIDO, Florencio et at. Diccionario de geografía urbana, urbanismo y ordenación del territorio. Madrid: Ariel, 2000, p. 384

\section{Cita del artículo}

MONGIL, D. Planificación urbana, regeneración urbana integral y participación ciudadana en Cataluña: balance y retos de futuro. Hábitat y Sociedad, 2012, № 4, p. 7391. <www.habitatysociedad.us.es $>$.

http://dx.doi.org/10.12795/HabitatySociedad.2012.i4.05 